\title{
Status of the development of diagnostic pressure gauges for the operation in ITER
}

\author{
Alexey Arkhipova, Andrea Scarabosio ${ }^{\mathrm{a}}$, Günter Haas ${ }^{\mathrm{a}}$, Felix Mackel ${ }^{\mathrm{a}}$, Jürgen Kolla ${ }^{\mathrm{a}}$, Hans Meister ${ }^{\mathrm{a}}$, \\ Horst Eixenberger ${ }^{\mathrm{a}}$, Oscar Paz ${ }^{\mathrm{b}}$, Fabien Seyvet ${ }^{\mathrm{c}}$, Santiago Terron ${ }^{\mathrm{c}}$ and Philip Andrew ${ }^{\mathrm{d}}$ \\ ${ }^{a}$ Max-Planck Institute for Plasma Physics, Boltzmannstraße 2, D-85748 Garching, Germany \\ ${ }^{b}$ Sgenia Solutions, C/Chile, 4 Edificio II, 28230 Las Rozas de Madrid, Madrid, Spain \\ ${ }^{c}$ Fusion for Energy, C/Josep Pla, $n^{\circ}$ 2, Torres Diagonal Litoral, Edificio B3, 08019 Barcelona, Spain \\ ${ }^{d}$ ITER Organization, Route de Vinon-sur-Verdon, CS 90 046, 13067 St. Paul Lez Durance Cedex, France
}

\begin{abstract}
The ITER Diagnostic Pressure Gauges (DPG) shall provide the measurement of the neutral gas pressure, which is an important parameter for basic control of the operation of the ITER machine as well as for input to physics models of the plasma boundary. The reference sensor is a hot cathode ionization gauge, which is able to operate in an environment with strong magnetic fields (up to 8 Tesla), strong and noisy background signals and fast pressure changes. In total 52 DPG sensor heads will be installed in 4 lower ports, 4 divertor cassettes and 2 equatorial ports. The DPG system is currently being developed by IPP in collaboration with F4E and Sgenia within the Framework Partnership Agreement FPA-364. As part of the system level design activities several different architectures of the DPG system, including gauge head and supporting electronic equipment, have been evaluated in detail with the aim to fulfil technical requirements imposed by the integration in ITER. Most important steps made towards the currently defined baseline design of the DPG system, which is based on ASDEX Pressure Gauge (APG) technology, are discussed in the present paper.
\end{abstract}

Keywords: ITER, pressure gauge, plasma diagnostics, system development, pressure measurement, RAMI.

\section{Introduction}

In ITER, plasma will interact with the first wall and the divertor and neutral gas will be continuously created and adsorbed also without puffing and pumping accompanied by a continuous exchange of energy and momentum. This non-linear interaction is important since it determines local plasma conditions and loads in the divertor area due to particle streaming, heat and impurity production. In this framework, the neutral pressure is a key variable in understanding and modelling the divertor performance [1].

A comparison of different diagnostics which are already in use for fusion applications such as Pirani gauge, Baratron, Penning gauge and different types of Hot-cathode ionization gauges, showed that ASDEX Pressure Gauge (APG) technology is most suitable for pressure measurements with regard to ITER requirements. The principal scheme of the APG sensor is shown in Fig. 1 [2].

The diagnostic provides measurement of the neutral gas density relying on impact ionization of the neutral gas by electrons. The cathode is a doped tungsten filament heated by the direct current and releases the electrons which are then accelerated by the control electrode (CE) also providing chopped operation by setting its voltage higher and lower than the filament potential to cancel large offsets and leakage currents. Afterwards electrons are further accelerated by the acceleration grid (AG) and reach the ionization volume where they collide and ionize the neutrals. The ion current $I_{i}$ and electron current $I_{e}$ are measured at the ion collector (IC) and the acceleration grid respectively.
Neutral gas pressure is then calculated by means of following equation [2]:

$$
P=\frac{I_{i}}{I_{e}-I_{i}} \frac{1}{d^{\prime}}
$$

where $d$ is the sensitivity of the gauge, which has to be determined in a calibration process for the pressure range and magnetic field strength that prevail in the fusion device. It should be noted that a linear arrangement of the electrodes (see Fig. 1) is required since the charged particles are forced to follow the magnetic field direction. The electrode and the filament planes have to be aligned perpendicular to the magnetic field lines. The APGs show a strong increase of sensitivity from $0 \mathrm{~T}$ to $1 \mathrm{~T}$. With further increase of the magnetic field the sensitivity stays almost constant. The APG design allows a variation of the magnetic field angle with respect to the gauge axis of about $+/-25^{\circ}$ [2]

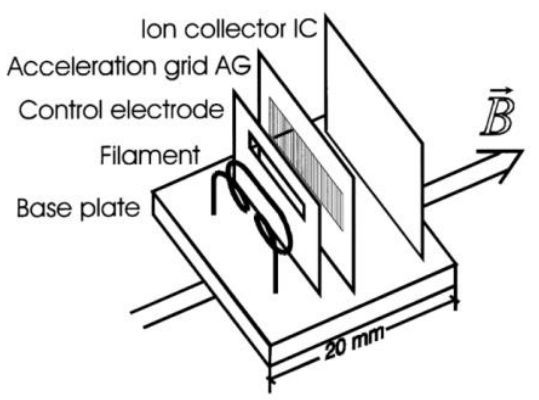

Fig. 1: Principal scheme of the APG sensor.

In present-day tokamaks and stellarators such as ASDEX Upgrade, DIIID, JET and W7-X [3] the APGs operate in various parts of the vacuum vessel, including the divertor region, at typical magnetic fields of 2-3.5 T. 
The sensors provide pressure measurement in a range of $10^{-3}-5 \mathrm{~Pa}$ with a time resolution of $1-2 \mathrm{~ms}[1,2]$. With a periodic in-vessel calibration the sensors are able to achieve a relative accuracy of $10-20 \%$. It should be noted that the length of the transmission $\operatorname{lines}^{1}$ is, so far, always below $50 \mathrm{~m}$.

For the operation in ITER major challenges for the diagnostic are posed by the harsh operational environment, namely: high magnetic field, high neutron fluencies and radiation on the sensors, dust, electromagnetic (EM) noise pickup, possibly high EM forces during disruption, high power of the electron cyclotron resonance heating (ECRH) and of the ion cyclotron resonance heating (ICRH). These effects together with the stringent accuracy and availability requirements in conjunction with the impossibility of repair or replacement of the sensor heads, except of planned replacement of the divertor cassettes with new Diagnostic Pressure Gauges (DPG), are driving design development for the sensors, electronics, cables and connectors.

Results of system level design (SLD) activities for the DPG sensor head and electronics as well as results of RAMI analysis of the complete DPG system are discussed in present paper.

\section{System level design activities for the DPG sensor head}

According to ITER requirements DPGs shall measure a pressure over a wide range of $10^{-4}-20 \mathrm{~Pa}$ with $20 \%$ of accuracy and $50 \mathrm{~ms}$ of time resolution. This means that ITER DPGs in contrast to current APG systems have to i) provide measurements at 3-4 times larger B-field, ii) measure 10 times lower and 4 times higher pressures with $20 \%$ accuracy, iii) maintain the required accuracy during the whole ITER life time with likely infrequent and/or incomplete calibrations and iv) attain a mean availability for the overall system of $98 \%$ with only one divertor sub-set replacement during the whole ITER life time.

Within the required pressure range the lower end is theoretically only limited by the signal-to-noise ratio of the ion current measurement. This, in turn, depends on i) the sensitivity of the sensor head, ii) the ability of the electronics to extract small signals buried in noise and iii) the noise level and frequency spectrum. The first two aspects can be dealt with the proper design but the latter is substantially unknown and only uncertain extrapolation and preventing measures are possible.

The upper pressure is limited by saturation effects. This depends intrinsically on the design. Laboratory tests have shown that the APG saturates with $\mathrm{B}>4 \mathrm{~T}$ at around $15 \mathrm{~Pa}$ [4] which is in contrast with the $8 \mathrm{~T}$ operation requirement in ITER. Note that a competition between the low and high pressure limits exists and one has to tailor the DPG system sensitivity in order to match both requirements simultaneously.

\footnotetext{
${ }^{1}$ Cables from the DPG head to the front-end electronics
}

Twenty percent relative accuracy over the pressure range of $10^{-4}-20 \mathrm{~Pa}$ is an achievable target similar to the industrial standard but challenged by the well-known ion gauge calibration drift with time. Some level of periodic in-vessel calibration must be assured in order to maintain the required accuracy during ITER life time although a single sensor will be designed to run for only a fraction of this time.

All mentioned challenges and needed validations have been taken into account during SLD activities and are carefully considered in the plan of prototype tests. Major design improvements of the APG system performed with the aim to fit ITER requirements are discussed in the sections below. Current design of the DPG sensor head is shown in Fig. 2.

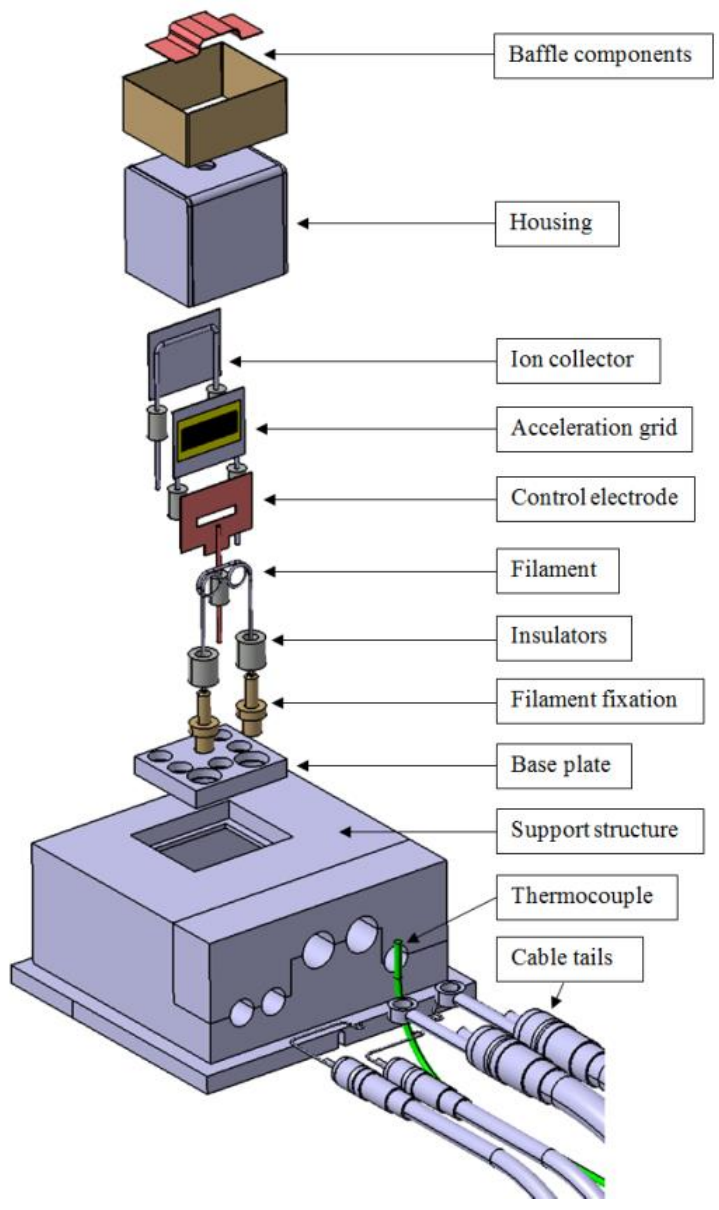

Fig. 2: Exploded view of the DPG head assembly.

\subsection{Investigation of design solutions to improve the operation of the DPG head}

Possibility to use a second filament: The filament is the subcomponent of the DPG head which is most prone to failure and for which it is difficult to estimate the lifetime. In case of a damaged or broken filament, a second filament may serve as a spare assuming that the other substructures remain intact. Unfortunately, this seems often not to be the case. It has to be considered that in case of an accident in ITER e.g. air ingress, the hot filament is likely to be destroyed under long cooling due to evaporation of tungsten oxide. The condensed vapor is likely to end up on the grids and insulators, 
strongly degrading their physical properties. Large filament deformation tends to damage the nearby control grid or second filament. As a consequence, the functionality of the whole DPG and all of its subsystems will be affected. A spare filament is not able to prolong DPG life time in this case. Therefore, it was decided to drop the implementation of a second filament.

Possibility to heat the filament with alternating current: Stress of the filament caused by $\vec{\jmath} \times \vec{B}$ forces is significantly lower when the filament is heated by alternating current. Therefore, thickness of the filament and, consequently, value of the heating current could be significantly reduced in comparison with the filaments heated by direct current. However, fatigue may become an issue. A thermo-mechanical assessment of AC heated filament demonstrated that it is possible to heat a thin filament of $0.2 \mathrm{~mm}$ radius with $\mathrm{AC}$ current amplitude of $2 \mathrm{~A}$. The frequency has to be high at about $45 \mathrm{kHz}$ in order to minimize peak stress at around $90 \mathrm{MPa}$ and deformation around $10^{-3} \mathrm{~mm}$. This, in turn, results in very high cycle numbers for which no reliable fatigue data is available in combination with high temperatures of $1900 \mathrm{~K}$. Thus, there is high degree of uncertainty whether the method of $\mathrm{AC}$ heating provides benefits compared to DC heating in terms of lifetime. Therefore, it was decided to use DC heating, which is a proven method.

Implementation of a thermocouple: The temperature of the divertor cassette and the vacuum vessel, where the DPG heads are mounted on, can vary significantly during full power operation of ITER. This results from the fact that the plasma is strongly heated and fusion reactions release additional energy. This power has to be dissipated in the surrounding structures and they will heat up despite strong cooling. It is estimated that the error in pressure reading can be more than $10 \%$ due to different temperatures during calibration and full power operation. A thermal simulation has shown that the temperature of the base plate centrally between the ion collector and the acceleration grid represents well the gas temperature in the ionization volume which is assumed to approach the average temperature of surrounding structures. Therefore, this measurement allows for the correction of the temperature variations in the sensor head affecting the measurement accuracy. Type $\mathrm{N}$ thermocouples are deemed as suitable candidates for temperature measurements up to $1200^{\circ} \mathrm{C}$ in the harsh ITER environment [5].

Implementation of a baffle: It has to be avoided that charged and fast particles reach the ionization volume and adversely affect the measurement. To prevent this a baffle is installed above the hole in the housing and forces those particles to collide with the walls. As a result they decelerate or recombine. The proposed design excludes the direct line of sight from the vacuum into the volume enclosed by the housing.

Cable tail versus plug-in connector: Consideration of the pros and cons for using of cable tails versus plug-in connectors showed that the cable tail solution is preferable since it provides more reliable electrical connections from the gauge head to the cables. In addition, the DPG heads are installed permanently in the machine and no replacement is foreseen. Therefore, the main advantage of plug-in connectors will not be used.

\subsection{Optimization of the electrode's geometry and potentials}

Simulations of the electron dynamics, collisions and ionization events, occurring during the operation of the DPG, have been performed by means of Monte Carlo code originally developed at IPP [6] which was improved and parallelized.

A consistent, systematic workflow to study the influence of relevant geometrical aspects and electric boundary conditions has been established with the aim to improve the sensitivity of the DPG with magnetic field and to avoid the saturation effect at pressure values below 20 Pa while keeping the minimum output current high enough for the device to be still functional. As a result of the parametric study a new optimized configuration of the DPG head including revised electrode potentials, grid positions relatively to the left edge of the base plate as shown in Fig. 1 and parameters of the acceleration grid has been identified (see Table 1).

Table 1: Parameters of the DPG head for reference and optimized configurations.

\begin{tabular}{|l|l|l|l|}
\hline \multicolumn{2}{|c|}{ Parameter $\backslash$ Configuration } & Reference & Optimized \\
\hline \multirow{4}{*}{ Voltages } & UFilament & $74 \mathrm{~V}$ & $100 \mathrm{~V}$ \\
\cline { 2 - 4 } & U Control_Electrode & $105 \mathrm{~V}$ & $160 \mathrm{~V}$ \\
\cline { 2 - 4 } & UAcceleration_Grid & $250 \mathrm{~V}$ & $195 \mathrm{~V}$ \\
\cline { 2 - 4 } & UIon_Collector & $0 \mathrm{~V}$ & $0 \mathrm{~V}$ \\
\hline \multirow{4}{*}{$\begin{array}{l}\text { Positions } \\
\text { meters }\end{array}$} & $Z_{\text {Filament }}$ & $5.65 \mathrm{~mm}$ & $5.65 \mathrm{~mm}$ \\
\cline { 2 - 4 } & $Z_{\text {Control_Electrode }}$ & $7.05 \mathrm{~mm}$ & $6.25 \mathrm{~mm}$ \\
\cline { 2 - 4 } & $Z_{\text {Acceleration_Grid }}$ & $9.25 \mathrm{~mm}$ & $8.5 \mathrm{~mm}$ \\
\cline { 2 - 4 } & $Z_{\text {Ion_Collector }}$ & $17.05 \mathrm{~mm}$ & $16 \mathrm{~mm}$ \\
\hline & Transparency & $80 \%$ & $40 \%$ \\
\cline { 2 - 4 } & Bar numbers & 28 & 56 \\
\cline { 2 - 4 } & Bar width & $0.1 \mathrm{~mm}$ & $0.15 \mathrm{~mm}$ \\
\cline { 2 - 4 } & Bar separation & $0.4 \mathrm{~mm}$ & $0.1 \mathrm{~mm}$ \\
\hline
\end{tabular}

The normalized output $\mathrm{I}_{\mathrm{i}} /\left(\mathrm{I}_{\mathrm{e}}-\mathrm{I}_{\mathrm{i}}\right)$ as function of the $\mathrm{H}_{2}$ gas pressure for different DPG configurations is presented in Fig. 3. It should be mentioned that due to simplified assumptions the simulation result with B-field tends to be more pessimistic (lower saturation pressure) than in reality. Thus, only relative changes are important here. A radically different behavior is found when comparing the standard settings to the optimized ones. As it is expected, and because of the much lower transparency of the acceleration grid and reduced residence times, the output in the optimized configuration is highly reduced as compared to the reference configuration (about two orders of magnitude lower). In contrast, the overall output shows a response closer to a linear behavior within the full pressure range, with calculated sensitivity slopes of $1.1 \mathrm{~Pa}^{-1}$ and almost irrespective of the magnetic field strength. Besides, it can be observed that the saturation region is greatly pushed up from few $\mathrm{Pa}$ to close to $20 \mathrm{~Pa}$ providing more confidence that the ITER requirement can be met. 

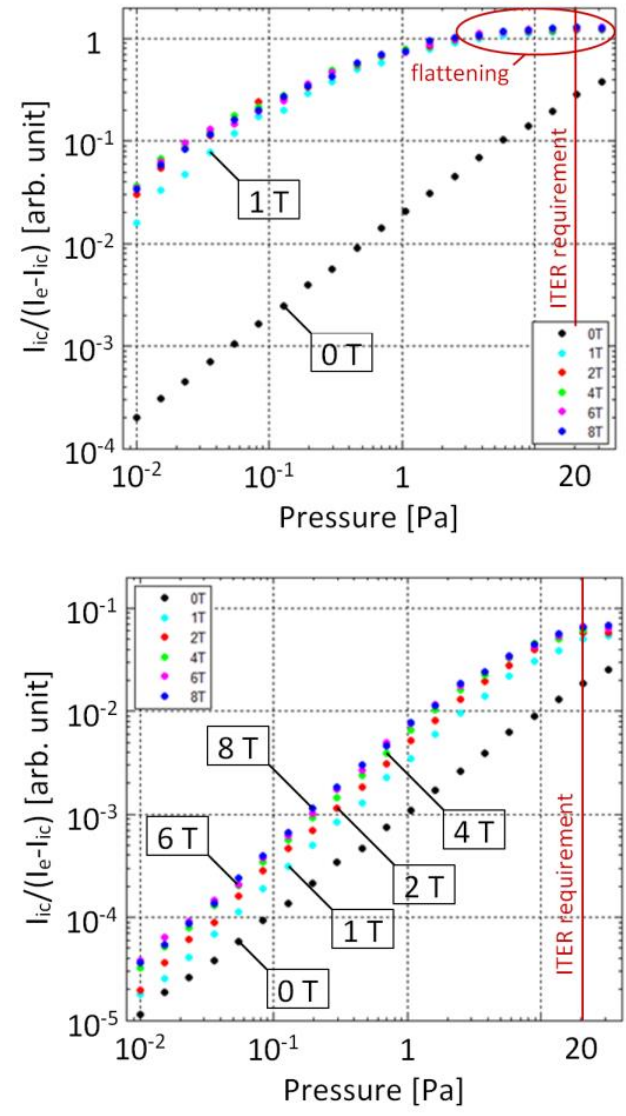

Fig. 3: Calculated normalized output $\mathrm{I}_{i} /\left(\mathrm{I}_{\mathrm{e}}-\mathrm{I}_{\mathrm{i}}\right)$ as function of the $\mathrm{H}_{2}$ gas pressure at the several magnetic field strength. Top panel corresponds to the reference configuration. Bottom panel is for the optimized configuration (note the different origin in ordinate values).

\section{System level design activities for the DPG electronics}

The main functions of the DPG control and measure electronics are i) to drive electric potentials of the DPG head electrodes, ii) to measure the ion and electron currents and iii) control the latter via a feedback loop. There are some specific difficulties and challenges in the realization of these functions related to the dimensions and a harsh environment of ITER.

Transmission lines connecting the DPG heads with front-end electronics will be several times longer than in the present-day experiments ${ }^{2}$, presenting also a multitude of junctions and connectors. As a consequence, the resistance and the capacitance of the cables will increase leading to a large distortion of the wave-form of the potentials applied to the electrodes. The electronics must compensate this effect in order to deliver the correct potential wave-form at the electrodes.

Another issue is the power dissipation by the long cables. Heating currents up to $20 \mathrm{~A}$ are required for the filament emission in steady state. In order to cope with power and temperature restrictions of the power supply, cabinet, connectors and cables themselves cables for

${ }^{2}$ Length of the transmission lines in ITER is expected to be up to $200 \mathrm{~m}$. filament heating with proper electrical resistivity must be chosen. A too large cable cross-section may, however, affect engineering and design restrictions of other ITER components like, for instance, feedthroughs and flanges, and, therefore, its compatibility must be carefully checked.

According to the operational experience of the APG system, external EM disturbances, generated by various components of fusion device or the plasma itself, may enter the system through unshielded electrical connections and reduce the signal-to-noise ratio to unacceptable levels. Therefore, proper shielding of each connector foreseen to be used in DPG system in ITER is required.

It should be mentioned that neutrons and gamma radiation may directly produce spurious ion currents which, in turn, may affect operation of the DPG system. A recently performed analysis showed that these effects are either acceptably small or could be efficiently eliminated by the chopped operation of the sensor. Although exact level and type of noise in ITER is not yet defined, a certain margin for noise reduction in the electric signals must be introduced in the design. This opportunity may come when considering the relatively slow measurement requirements of $50 \mathrm{~ms}$ compared with the present 1-2 ms. Extra signal filtering or smoothing may help in achieving the required signal-to-noise ratio over the whole pressure range in case of excessive noise.

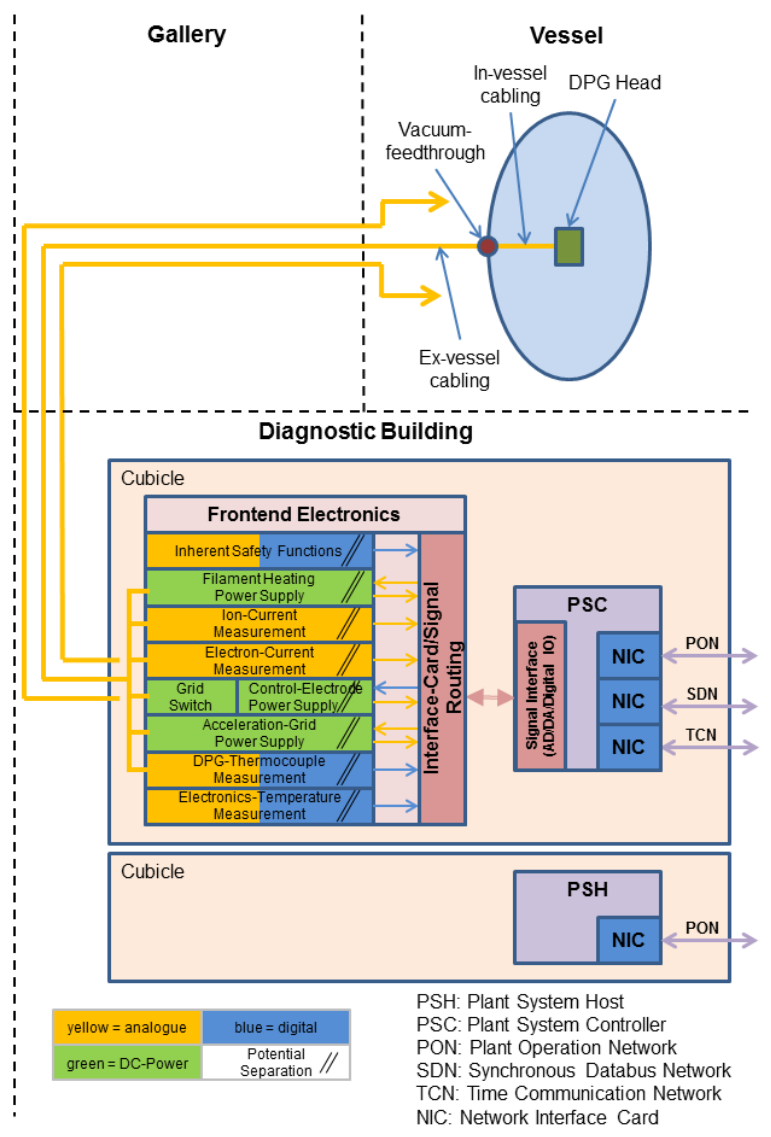

Fig. 4: Block diagram of the selected DPG electronics architecture. 
Several architecture options of the DPG electronics have been evaluated as the part of SLD activities. Proposed architectures differ in the distribution of the electronic components within ITER buildings. The choice of the DPG electronics architecture was driven by the results of long cable tests, according to which stable operation of the DPG was proven with a length of transmission lines up to $200 \mathrm{~m}$ and a chopping frequency of at least $1 \mathrm{kHz}$. Thus, it was recommended to accommodate all electronic components in the diagnostic building where in respect with tokamak building i) space requirements are less demanding, ii) environment in terms of magnetic field and radiation is less harsh and iii) accessibility to the components is better. Block diagram of the selected DPG electronics architecture is shown in Fig. 4.

\section{Results of RAMI analysis}

In frame of the SLD process RAMI analysis of the complete DPG system including all sensor heads and electronic sets as well as cables, connectors and feedthroughs has been performed in line with the ITER RAMI Program. Calculations showed that the mean system availability over 20 years of ITER lifetime for recommended configuration when any 8 of 52 sensors are in operation is $99.9 \%$. This value is higher than the $98 \%$ requirement for the pressure measurement availability and, therefore, this system configuration is a valid operational regime for the DPG diagnostic. The availability requirement will not be obtainable for maximal system configuration when any 16 out of 52 sensors are in operation. Nonetheless, the mean availability in this case is $95.1 \%$ which is still high. Due to the margin with the recommended configuration it might be possible to operate in the maximal configuration for a small fraction of pulses.

It should be noted that according to the results of the performed RAMI analysis operational plan for the DPG system in ITER was modified and the number of electronic sets was reduced from 24 to 16 .

\section{Conclusion and future work}

The baseline design for the DPG head and supporting front-end electronics has been defined applying the SLD approach. Even though design solutions developed during the SLD phase have been supported by dedicated calculations and modelling activities, the complete validation of the DPG system design will be based on the results of the planned prototype testing, which will include tests on measurement, reliability and engineering performances.

Tests on the measurement performance will comprise investigations of the measurement uncertainty and reproducibility with special regard to the upper pressure limit. Electronic components are planned to be tested both individually and as integrated system including the operation of the DPG head in vacuum.

Reliability performance tests are planned to demonstrate the reliability compliance among a number of preselected filament materials. The operational margin shall be assessed by identifying the minimum current required to produce sufficient electron emission over a long period of time and the maximum current in a high magnetic field causing structural damage to the filament. Subsequent tests shall provide information on the reliability of the filament in repeated start-ups and long term operation in a magnetic field. Despite the fact that ITER conditions cannot be fully simulated, the experiments can give a more reliable estimate of the filament lifetime and hence a more precise RAMI analysis. On the electronics side, the system has to comply with several standards which are described in the ITER Electrical Design Handbook.

The temperature distribution of the DPG head and of the filament under various operating conditions will be measured as well. These tests aim towards ensuring that the thermocouple installed in the baseplate allows the expected correction of the pressure measurement, under varying environmental temperatures. In addition, these tests will allow to benchmark finite element simulations of the DPG head, which are particularly relevant in the case of the filament thermo-mechanical behavior.

\section{Acknowledgments}

This work was partly supported by Fusion for Energy under the Specific Grants F4E-FPA-364-SG03 and F4EFPA-364-SG04. The views expressed in this publication are the sole responsibility of the authors and do not necessarily reflect the views of the Fusion for Energy and the ITER Organization.

Neither Fusion for Energy nor any person acting on behalf of Fusion for Energy is responsible for the use, which might be made of the information in this publication.

\section{References}

[1] A. Scarabosio et al, Journal of Nuc. Mat. 390-391, (2009) 494-497

[2] G. Haas, H.S. Bosch, Vacuum 51 (1998) 39-46

[3] H. Nielson (Ed.), Magnetic Fusion Energy: From Experiments to Power Plants, Woodhead Publishing Series in Energy: Number 99, Elsevier, (2016), ISBN: 978-0-08100315-2

[4] A. Scarabosio, G. Haas, in: AIP Varenna Conference Proceedings, vol. 998 (238), 2008

[5] N. A. Burley, Nicrosil-Nisil Type N Thermocouples, Measurements \& Control, April (1989) 130-133

[6] A. Scarabosio, P. Sauter, G. Haas, Nucl. Instr. Meth. Phys. Res. A 623 (2010) 667-671 